\title{
Antibiotic Resistance Pattern of Shigella spp. Among Gastroenteritis Patients at Tertiary Care Hospital in Pokhara, Nepal
}

\author{
Kabi Thapa1,2, Balkrishna Bhattachan2,3*, Raja Ram Gurung, Archana Katuwal1, \\ Jagat Bahadhur Khadka² \\ ${ }^{1}$ Department of Microbiology of Tri-Chandra Campus, Kathmandu, Nepal \\ ${ }^{2}$ Western Regional Hospital, Pokhara, Nepal \\ ${ }^{3}$ Siddhi Memorial Hospital, Bhaktapur, Nepal \\ ${ }^{4}$ Deurali-Janta Pharmaceuticals Pvt. Ltd., Kathmandu, Nepal
}

\begin{abstract}
Shigellosis, a disease caused by Shigella species. It is a major public health problem in developing nations like Nepal, where communities having poverty; poor sanitation, personal hygiene, and water supplies. The main aim of our study is to isolate and identify Shigella spp. from gastroenteritis patients and to find out its drug resistance pattern.

A cross-sectional study was carried out based on routinely attending outpatients and inpatients. A total of 225 stool samples collected from gastroenteritis patients were processed from 20 April to 24 September 2014 in Western Regional Hospital, Pokhara, Nepal. Standard microbiological procedures were followed for the isolation of Shigella spp. After that slide agglutination kit method was used for identification of Shigella spp. Finally, Kirby-Bauer disc diffusion method was done for an antimicrobial resistance test.

Of the total 225 gastroenteritis patients, 133 were detected as bacterial positive cases. Among positive cases, Shigella spp. was identified in $10.5 \%$. Age wise, an infection rate of Shigella in patients $<15$-years old was found higher i.e. $7.3 \%$ than in patients $\geq 15$ years old i.e. $4.5 \%$ with the $(\mathrm{p}=0.432)$ at $95 \%$ CI. The infection rate of S. dysenteriae, S. flexneri, and S. sonnei was detected in $28.6 \%, 57.1 \%$, and $14.3 \%$ respectively. For the antimicrobial test, eight types of antibiotics were used. The most resistance pattern of isolated Shigella spp. was found in nalidixic acid, and co-trimoxazole $92.8 \%$ followed by ampicillin $64.3 \%$ and ciprofloxacin $42.8 \%$ etc.

Our study reported that endemicity of Shigellosis with S. flexneri is the predominant group in gastroenteritis patients. This finding suggests that co-trimoxazole, nalidixic acid, ciprofloxacin and ampicillin should not be used experimentally as first-line drugs for shigellosis treatment.

Keywords: Shigella, Antibiotic Resistance Pattern, Shigellosis, Gastroenteritis, Tertiary Care Hospital, Nepal

*Corresponding author

Email: balkrishabhattachan@gmail.com
\end{abstract}

\section{Introduction}

Shigellosis is caused by members of the bacterial genus Shigella. It is a severe and occasionally life-threatening gastrointestinal infection. Worldwide, Shigella spp. is the most common cause for acute and bloody diarrhea (dysentery). They are also responsible for a significant proportion of the burden of morbidity and mortality associated with diarrheal disease [1, 2]. The incidence of shigellosis in developing countries is nearly 20 times more than in developed countries [3]. Annually, it is estimated that there are 125 million infections and 14,000 deaths due to shigellosis in Asia. [4].

The first emerging antimicrobial studies conducted in the 1950s, reported multiple drug resistance transmitted by plasmids among Shigella spp. from many countries $[5,6]$. As a result of the considerable global burden, low infectious dose, clinical severity, and frequent reports of emerging antimicrobial resistance against first-line and, more recently, secondline therapies [7-9]. Over $70 \%$ of Shigella isolates were resistant to two or more drugs including ampicillin and co-trimoxazole during 2002 to 2007 in India [10]. Reports from Indonesia, Bangladesh, Malaysia and Nepal, showed an increasing prevalence of Shigella isolates that are resistant to multiple drugs like trimethoprim-sulphamethoxazole, ampicillin, nalidixic acid and tetracycline [1116].

Shigellosis is associated with significant morbidity and mortality among the pre-school children. It is caused by any one of the four species of Shigella, namely Serotype A - S. dysenteria, Serotype B - S. flexneri, Serotype C - S. 
sonnei, and Serotype A - S. boydii, and the classification is based upon the serological antigen [17]. The frequencies of S. flexneri, $S$. sonnei, S. boydii, and S. dysenteriae are $16.0 \%$, $7.0 \%, 2.0 \%$, and $1.0 \%$ in developed countries and $60.0 \%, 15.0 \%, 6.0 \%$, and $6.0 \%$ in developing countries, respectively [18]. In Nepal, Shigella spp. was detected in $13.6 \%$ of stool samples examined at Nepalgunj Medical College and Teaching Hospital, Banke, between September 2011 and April 2013 [19]. The current study was carried out in stool specimens collected from gastroenteritis patients at a Tertiary Care Hospital in Pokhara, Nepal. The main aim of this study is to isolate and identify Shigella spp. from gastroenteritis patients and find out its antimicrobial resistant pattern by modified Kirby-Bauer disk diffusion method.

\section{Materials and Methods Study design and setting}

A cross-sectional study was conducted in 225 stool samples collected during 20 April to 14 September 2014. Clinical specimens of gastroenteritis patients were examined which is referred by a physician. The patient's symptoms like diarrhea, abdominal pain, vomiting, reddish \& watery stool; age, gender etc. were also recorded. Both outpatient and in patients with acute gastroenteritis but not undertaking any antibiotics were taken as subject from Western Regional Hospital.

\section{Sample collection}

One gram of fresh stool sample was collected in a clean and sterile screw-capped plastic container. The collected samples were labeled properly with the patient's name, age, sex, address, and date. These samples were processed immediately following Standard Operating Procedures (SOPs) of microbiology in the laboratory of Western Regional Hospital, Pokhara, Nepal. If there was a delay in processing freshly passed stool sample within 2 hours, the specimens were kept in a refrigerator at $2-8^{\circ} \mathrm{C}$.

\section{Macroscopic examination}

Microscopic physical examination of stool sample was done for the color, consistency (formed, semi-formed, unformed), presence of blood, mucus or pus.

\section{Isolation and identification of Shigella on Culture method:}

$0.1 \mathrm{ml}$ of stool sample were inoculated in MacConkey (MA), Xylose Lysine Deoxycholate (XLD) and Salmonella-Shigella (SS) agar (Himedia Lab. Pvt. Ltd. India) and incubated at $3^{\circ} \mathrm{C}$ for 24 hours. The suspected colonies were grouped as Gram Positive and Gram Negative by Gram's staining reaction. Biochemically, Shigella are found Indole negative, Citrate negative, Urea negative and Hydrogen Sulphide negative in TSI agar's slant and nonmotile [14].

\section{Identification of Shigella spp. in slide agglutination test}

Shigella spp. was further tested by type-specific antisera of group A, B, C and D Shigella antisera in a serological kit (Denka Seiken Co. LTD. 3-42, Nihonbashi Kayabacho, Chuo-ku, Tokyo, Japan). Agglutination appeared on (Group A) monovalent, (Group B) polyvalent, (group C) polyvalent and (Group D) polyvalent in Kit, were identified for $S$. dysenteriae, S. flexneri, $S$. boydii and $S$. sonnei respectively.

\section{Antibiotic susceptibility test}

Antibiotic susceptibility tests of different clinical isolates against various antibiotics were performed by modified Kirby-Bauer disk diffusion method in Mueller Hinton Agar (HiMedia Laboratories, India) according to Clinical Laboratory Standards Institute (CLSI) guidelines [20]. The antibiotics used for analysis were ampicillin, azithromycin, ciprofloxacin, co-trimoxazole, ceftriaxone, gentamycin, and nalidixic acid. The diameters of inhibition of zone for Shigella were compared with strains Escherichia coli ATCC 25922 (National Committee for Clinical Laboratory Sciences, Document M100-56: Sixth informational Supplement).

\section{Statistical analysis}

Pearson's Chi-Square test was used to determine the significant association of dependent variable by using Win-pepi Software (Copyright J. H. Abrmson, 23 Aug 
2016 Version 11.65) with the p-value $>0.05$ is considered as significant at $95 \% \mathrm{CI}$.

\section{Results}

Of the total 225 gastroenteritis patients, 133 were as detected bacterial positive cases (Grampositive $=54$ and Gram-negative $=79$ ). Among bacterial positive cases, Shigella spp. was detected in $10.5 \%(14 / 133)$. The infection rate of S. flexneri, S. dysenteriae, and S. sonnei were detected in $57.1 \%(8 / 14), 28.6 \%(4 / 14)$ and $14.3 \%(2 / 14)$ respectively (Figure 1).

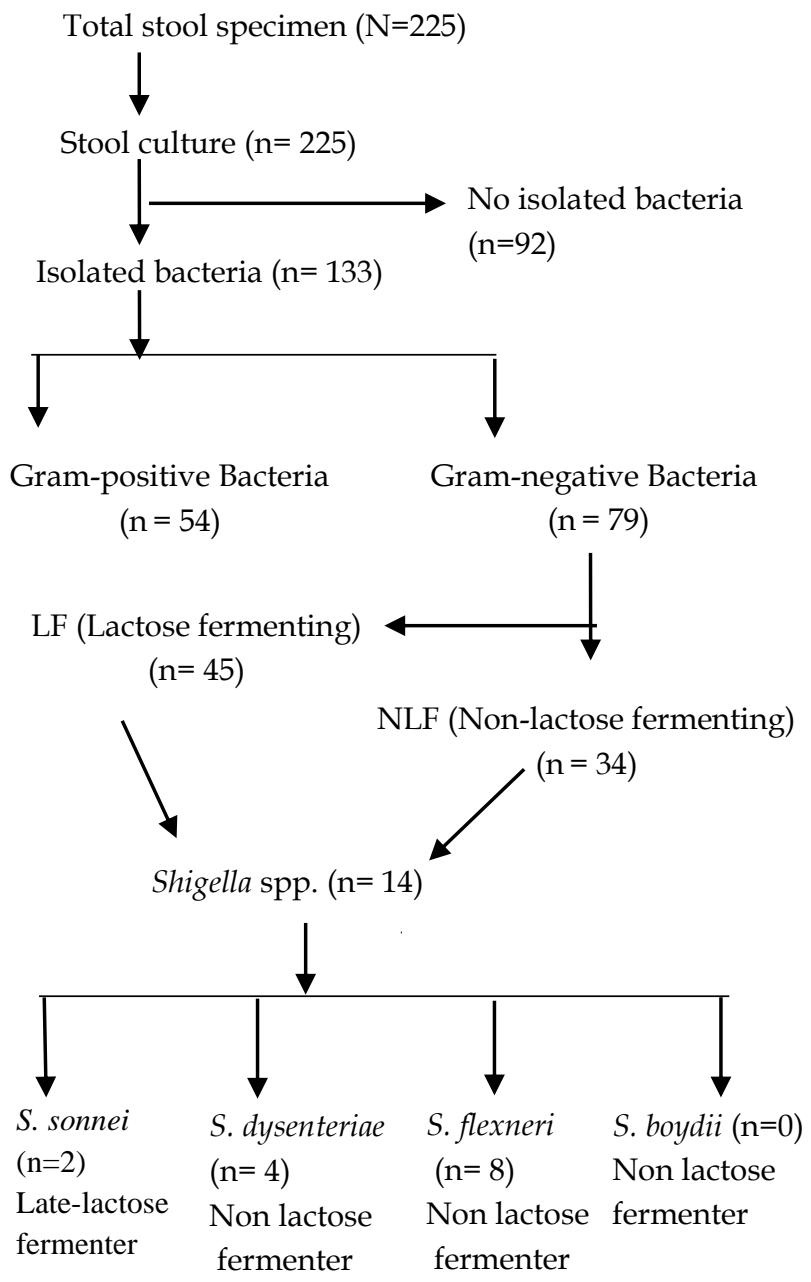

Figure 1. Flow chart for the isolation and identification of Shigelle isolates.

\section{Sex wise distribution of all positive cases in different species of Shigella in gastroenteritis patients:}

Among bacterial positive cases, male patients were 129 and female patients were 96 in the ratio of 1.3:1. In male patients, infection rate of S. flexneri was the highest i.e. $42.8 \%(6 / 14)$, followed by $S$. dysenteriae $7.1 \%(1 / 14)$ and $S$. sonnei $7.1 \%(1 / 14)$. Whereas in female patients, the highest rate of infection was observed for $S$. dysenteriae, i.e. $21.1 \%(3 / 14)$, followed by $S$. flexneri $14.3 \%(2 / 14)$ and S. sonnei $7.1 \%(1 / 14)$ were depicted in figure 2.

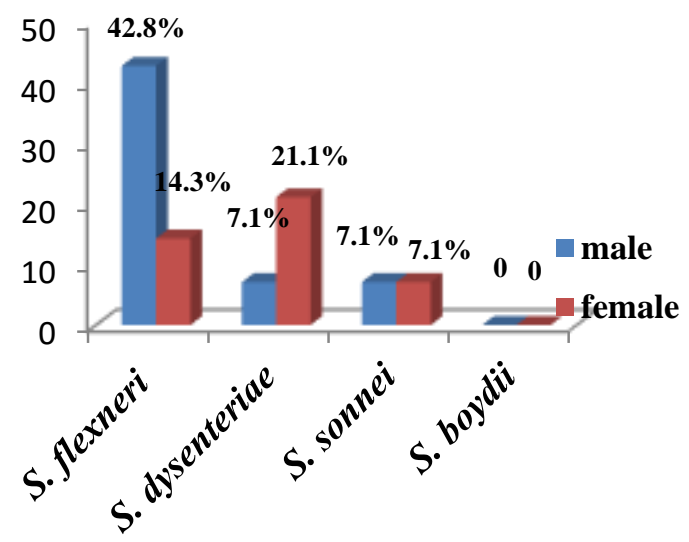

Figure 2: Sex wise distribution of all positive cases in different species of Shigella in gastroenteritis patients.

\section{Age-wise distribution of gastroenteritis patients with Shigella and its infection:}

Infection rate for Shigella was $7.3 \%(10 / 137)$ and $4.5 \%(4 / 88)$ in patient $<15$ years old and $>15$ years old respectively, confirming that infection rate for Shigella spp. is higher in children patients than in patients elder than 15 years old with the $p$-value $(p=0.432)$ at $95 \%$ CI (Table 2$)$.

\section{Antibiotic resistance wise distribution of gastroenteritis patients with Shigella and its species infection:}

In antibiotic resistance test, the most resistance pattern of isolated Shigella were found in nalidixic acid and co-trimoxazole $92.8 \%$ (13/14) followed by ampicillin $64.3 \% \quad(9 / 14)$ and ciprofloxacin $42.8 \%(6 / 14)$, detail description of Shigella spp. were shown in Table 3.

\section{Discussion}

Worldwide, acute gastrointestinal infections including diarrhea are among the leading causes of morbidity and mortality among children, particularly in underdeveloped countries [21]. Poor access to safe water, inadequate sanitary conditions, lower literacy rate, and unavailability of healthcare facilities in the remote area are the major factors 
Table 2: Age with isolated Shigella spp.; S. flexnari, S. dysenteriae, and S. sonnei in patients of gastroenteritis

\begin{tabular}{lllllllll}
\hline Characteristics & & $\begin{array}{l}\text { Total stool } \\
\text { sample } \\
(\mathrm{n}=225)\end{array}$ & $\begin{array}{l}\text { Shigella } \\
(\mathrm{n}=14)\end{array}$ & P value & $\begin{array}{l}\text { S. flexnari } \\
(\mathrm{n}=8)\end{array}$ & $\begin{array}{l}\text { S. dysenteriae } \\
(\mathrm{n}=4)\end{array}$ & $\begin{array}{l}\text { S. sonnei P-value } \\
(\mathrm{n}=2)\end{array}$ \\
\hline \multirow{2}{*}{ Age (year) } & $<15$ & 137 & No. $(\%)$ & & No. $(\%)$ & No. $(\%)$ & No. $(\%)$ \\
\cline { 3 - 7 } & & $10(7.3)$ & $>0.05$ & $5(3.6)$ & $3(2.1)$ & $2(1.4)$ & $>0.05$ \\
& 215 & 88 & $4(4.5)$ & & $3(3.4)$ & $1(1.3)$ & $0(0.0)$ \\
\hline
\end{tabular}

Table 3 Antibiotic Resistance pattern of Shigella and its species

\begin{tabular}{lcccc} 
Table 3 Antibiotic Resistance pattern of Shigella and its species \\
\hline Antibiotics & S. flexnari $(\mathrm{n}=8)$ & S. dysenteriae $(\mathrm{n}=4)$ & $\begin{array}{c}\text { S. sonnei } \\
(\mathrm{n}=2)\end{array}$ & Total $(\mathrm{n}=14)$ \\
\hline & No. $(\%)$ & No. $(\%)$ & No. $(\%)$ & No. $\%$ \\
\cline { 2 - 5 } Ampicillin $(10 \mu \mathrm{m})$ & $4(50.0)$ & $3(75.0)$ & $2(100.0)$ & $9(64.3)$ \\
Azithromycin $(10 \mu \mathrm{m})$ & $1(12.5)$ & $0(0.0)$ & $0(0.0)$ & $1(7.1)$ \\
Ciprofloxacin $(5 \mu \mathrm{m})$ & $2(25.0)$ & $3(75.0)$ & $1(50.0)$ & $6(42.8)$ \\
Ceftriaxone $(30 \mu \mathrm{m})$ & $2(25.0)$ & $2(50.0)$ & $0(0.0)$ & $4(28.6)$ \\
Co-trimoxazole $(25 \mu \mathrm{m})$ & $8(100.0)$ & $3(75.0)$ & $2(100.0)$ & $13(92.8)$ \\
Gentamycin $(10 \mu \mathrm{m})$ & $2(25.0)$ & $0(0.0)$ & $0(0.0)$ & $2(14.3)$ \\
Nalidixic acid $(30 \mu \mathrm{m})$ & $7(87.5)$ & $4(100.0)$ & $2(100.0)$ & $13(92.8)$ \\
\hline
\end{tabular}

predisposing diarrheal illness among developing countries [22]. Shigella spp. was detected in $10.5 \%$, among which, infection rate of S. flexneri, S. dysenteriae, and S. sonnei was detected in $57.1 \%, 28.6 \%$, and $14.3 \%$, respectively in gastroenteritis patients. S. boydii could not identify, this might be due to small sample size or error in the kit method in diarrheal patients, or might be used by kit method rather than molecular methods. In the previous study, it was reported that $S$. flexneri $(43.2 \%)$ is the predominant of the four species followed by S. dysenteriae (41.5\%), S. boydii $(7.6 \%)$ and S. sonnei $(7.6 \%)$ in Nepal [19]. Similarity, the prevalence of $S$. flexneri is identified in $42.0 \%$ of isolates, while $S$. dysenteriae in $27.5 \%$, S. boydii in $21.7 \%$ and $S$. sonnei in $8.7 \%$ in Eastern Nepal [18]. In western Nepal, S. flexneri, S. dysenteriae, S. boydii, and S. sonnei were accounted respectively for $43.1 \%$, $27.7 \%, 21.5 \%$, and $7.7 \%$ of the total number of Shigella isolated [14].

Infection rate for Shigella spp. was higher (7.3\%) in gastroenteritis patients of $<15$ years old than in patients $\geq 15$ years old $(4.5 \%)$ which is not statistically significant ( $p=0.432)$. This finding is consistent with the study done by Khan et al and Shakya et al found Shigella $42.0 \%$ and $38.4 \%$ and $30.1 \%$ respectively [14, 18, 32]. The reported high prevalence of Shigella spp. from children aged 1-10 years, compared to the other age groups. They also reported high prevalence of Shigella spp. from male patient compared to the female [14, 32].

Shigellosis or severe bacillary dysentery is a disease of public health importance because it is associated with increased mortality and morbidity especially among the children of developing countries [23]. The seasonal tendency of shigellosis was summer-monsoon [24]. Our study was conducted in summermonsoon (April to September); when school going children may get dysentery and diarrhea due to unsafe water like rainfall, flood and drinking contaminated water. On the other hand the no. of male cases with the pathogen exposer were high may be due to they go out from home more frequently for playing, eating purpose that compares to female.

For antibiotic resistance pattern, eight types of antibiotics were used. Nalidixic acid and cotrimoxazole $(92.8 \%)$ was found to be the most resistant antibiotics in isolated Shigella spp. followed by ampicillin (64.3\%) and ciprofloxacin $(42.8 \%)$. Among isolated Shigella Spp., almost all of the $S$. flexneri is most resistant to co-trimoxazole $(100.0 \%)$ while $S$. dysenteriae is resistant to nalidixic acid (100.0\%). In addition, most of the S. sonnei was found 100.0 $\%$ resistant in ampicillin, nalidixic acid, and cotrimoxazole. In a study, resistance rate of 
Shigella spp. for nalidixic acid was 95.6\%, ampicillin $85.5 \%$, co-trimoxazole $82.6 \%$, gentamicin $24.6 \%$, ofloxacin $21.7 \%$ etc. [19]. $33 \%$ of the total Shigella studied was found multi drug resistant (MDR) i.e. they showed resistance to 3 or more antibiotics. However, none of the Shigella spp. was resistant to azithromycin and ceftriaxone. Ciprofloxacin resistance was seen only among Shigella dysenteriae strains [14]. Shigella spp. resistant to nalidixic acid (95.4\%); ampicillin (84.6\%) cotrimoxazole $(81.5 \%)$ and ciprofloxacin $(46.2 \%)$ were detected in Nepal [16]. Over the past decades, it has been reported that a significant number of Shigella spp. isolates resistant to normally prescribed drugs [25]. In the early 1990s, many isolates of Shigella spp. were susceptible to norfloxacin, nalidixic acid, gentamicin and furazolidone [26, 27]. However, in the late 1990s, most Shigella isolates showed an increased resistance to these antibiotics [28, 29] but most were susceptible to ciprofloxacin [30]. Nowadays, alternative drugs such as the third generation cephalosporins are being commonly used. Although, the present study shows that Shigella strains are rapidly acquiring resistance to these substituted drugs as well. The emergence of plasmid-borne resistance to cephalosporins is another reason which further reduces the therapeutic option for the treatment of shigellosis [19]. Shigella spp. is highly necessary to start a prompt and rational antibiotic regimen to minimize the clinical effects of severe dysentery and its complications [31].

We could not able to perform confirmatory tests like other biochemical tests, molecular level test like PCR, gel electrophoresis due to lack of resources and additional clinical patients issues like clinical complication and informed consents. Moreover, the study was limited to the single hospital so the results do not represent a broad population.

\section{Conclusions}

Our findings also revealed that endemicity of Shigellosis with $S$. flexneri is the predominant strain in gastroenteritis patients. To prevent school going children from Shigellosis they should keep away from untreated water. As shown by antibiotic resistant pattern, cotrimoxazole, nalidixic acid, ciprofloxacin,and ampicillin should not use experimentally as first-line drugs for the treatment of shigellosis. Frequent analysis of resistance pattern and periodic reporting of Shigella spp. is necessary for Shigellosis therapy. In addition, continuous cultural surveillance of multidrug-resistant test of Shigella spp. is necessary to know changing the time-to-time resistant pattern of its serogroup. Isolation and sensitivity testing for Shigella spp. should be done regularly. Monitoring of emergence of resistance is highly recommended.

\section{Ethical approval and consent to participant}

All Shigella strains were routinely collected in the microbiology laboratory. No patient-related data were collected. Ethical approval was therefore not required. The study was laboratory-based basic science study.

Written informed consent was taken from all participating patients or from guardian on the behalf of their children.

\section{Consent for publication}

Not applicable

\section{Availability of data and materials}

All supplementary files, data generated and analyzed during this study will be made available as per request to co-author.

\section{Source of support:}

None

\section{Conflict of interest:}

None declared

\section{Author's contributions}

KT and AK designed the study. KT collected sample at Western Regional Hospital. KT and AK performed an investigation and recorded the laboratory findings with the validation. JBK supervised and provide methodology for the study. RRG and BB administered the project, reviewed literature, and written original manuscript; curated data to perform statistical 
data analysis and data interpretation. RRG helped in review and revision of the draft by compiling, formatting, editing and writing the final version of the article. Thus, all authors made a substantial contribution to the study. All of them read and approved the final manuscript.

\section{Acknowledgements}

We are indebted to staff and volunteer at Western Regional Hospital and Tri-Chandra college's friends who help us continuously. Without their supports, we could not conduct this research.

\section{References}

1. Thapar N, Sanderson IR. Diarrhea in children: an interface between developing and developed countries. Lancet. 2004 363: 641653. PMID: 14987892

2. Kotloff KL, Nataro JP, Blackwelder WC, Nasrin $\mathrm{D}$, Farag TH, et al. Burden and aetiology of diarrhoeal disease in infants and young children in developing countries (the Global Enteric Multicenter Study, GEMS): a prospective, case-control study. Lancet. 2013 382: 209-222. DOI: 10.1016/S0140-6736(13) 60844-2 PMID: 23680352

3. Parija SC: Textbook of Microbiology and Immunology (2nd edition Elsevier). A division of Reed Elsevier India Private Limited. 2012 281285.

4. Bardhan P, Faruque A, Naheed A, Sack DA: Decrease in shigellosis-related deaths without Shigella spp.-specific interventions, Asia. Emerg Infect Dis. 2010, 16: 1718-1723. doi: 10.3201/eid1611.090934 PMID: 21029529

5. Brooks GF, Carroll KC, Butel JS, Morse SA: Jawetz, Melnick, \& Adelberg's Medical Microbiology. 24th ed. New York: McGrawHill. 2007 224-226.

6. Njunda AL, Assob JC, Nsagha DS, Kamga HL, Awafong MP, Weledji EP: Epidemiological, clinical features and susceptibility pattern of shigellosis in the Buea Health District, Cameroon. BMC Res Notes. 2012 5: 54.

7. DuPont HL, Levine MM, Hornick RB, Formal SB: Inoculum Size in Shigellosis and Implications for Expected Mode of Transmission. J Infect Dis. 1989 159: 11261128. PMID: 2656880

8. Gu B, Cao Y, Pan S, Zhuang L, Yu R, et al.: Comparison of the prevalence and changing resistance to nalidixic acid and ciprofloxacin of Shigella between Europe-America and Asia-Africa from 1998 to 2009. Int $J$ Antimicrob Agents. 2012 40: 9-17. Doi: 10.1016/j.ijantimicag.2012.02.005
9. Vinh H, Baker S, Campbell J, Hoang NVM, Loan HT, et al.: Rapid emergence of third generation cephalosporin resistant Shigella spp. in Southern Vietnam. J Med Microbiol. 2009 58: 281-283. doi: 10.1099/ jmm.0.0029490 PMID: 19141753

10. Srinivasa H, Baijayanti M, Raksha Y: Magnitude of drug resistant shigellosis: A report from Bangalore (Brief Communication). Indian J Med Microbiol. 2009 27: 358-360.

11. Subekti D, Oyofo BA, Tjaniadi P, Corwin AL, Larasati $W$, Putri $M$ et al: Shigella spp. surveillance in Indonesia: the emergence or reemergence of $S$. dysenteriae. Emerg Infect Dis. 2001 7: 137-140.

12 Shahid NS, Rahaman MM, Haider K, Banu H, Rahman N: Changing pattern of resistant Shiga bacillus (Shigella dysenteriae type 1) and Shigella flexneri in Bangladesh. J Infect Dis. 1985 152: 1114-119.

13. Thong KL, Hoe CH, Koh YT, Yasim RM: Prevalence of multidrug-resistant Shigella isolated in Malaysia. J. Health Popul Nutr. 2002 20(4): 356-358.

14. Khan S, Singh P, Ansari M, Asthana A: Isolation of Shigella species and their resistance patterns to a panel of fifteen antibiotics in mid and far western region of Nepal. Asian Pac J Trop Dis. 2014 4(1):30-34.

15. Hamata OP, Chinsembu KC: Use of selective media and colony polymerase chain reaction to isolate Shigella from water catchments in Namibia.J Res Microbes. 2012 1(1): 44-50.

16. Talukder KA, Azmi IJ: Population genetics and molecular epidemiology of Shigella species. In: Faruque SM, editor. Foodborne and waterborne bacterial pathogens epidemiology, evolution and molecular biology. Caister Academic Press. 2012 :63-76.

17. Marrdanesh J, Poor SA and Afrugh P: Prevalence of Shigella spp. and antimicrobial resistance pattern of isolated strains from infected pediatrics in Tehran. Int J EntricPathog. 2013 1(1).

18. Khan S, Singh P, Asthana A, and Ansari M: Magnitude of drug resistant shigellosis in Nepalese patients. Iranian J Microbiol. 2013 5: 334-338.

19. Kansakar P, Malla S, Ghimire GR: Shigella isolates of Nepal: changes in the incidence of Shigella subgroups and trends of antimicrobial susceptibility pattern. Kathmandu Uni Med J. 2007 5(17): 32-37.

20. Clinical and Laboratory Standards Institute: Performance standards for antimicrobial susceptibility testing; Twenty Fifth Information Supplement, In. Wayne. USA: 2016: 98-101.

21. Centers for Disease Control and Prevention. Shigella-Shigellosis. Centers for Disease Control and Prevention, Atlanta, Ga, USA 2016. 
http://www.cdc.gov/shigella/generalinformation.html.

22. Sangeetha AV, Parija SC, Mandal J, and Krishnamurthy S: Clinical and microbiological profiles of Shigellosis in children. J of Hlth, Pop and Nutr. 201432 (4): 580-586.

23. Gupta S, Mishra B, Muralidharan S, Srinivasa $\mathrm{H}$ : Ceftriaxone resistant Shigella flexneri, an emerging problem. Indian J of Med Sci. 2010 64 (12): 553-556.

24. Bennish ML, Salam MA, Hossain MA, Myaux J, Khan EH, Chakraborty J, et al: Antimicrobial resistance of Shigella isolates in Bangladesh, 1983-1990: increasing frequency of strains multiply resistant to ampicillin, trimethoprim-sulfamethoxazole, and nalidixic acid. Clin Infect Dis. 1922 14: 1055-1060.

25. Jesudason MV: Shigella isolation in Vellore, South India (1997-2001). Indian J Med Res. 2002 115: 11-13.

26. Thapa BR, Ventkateswarlu K, Malik AK, Panigrahi D. Shigellosis in children from North India: a clinic pathological study. J Trop Pediatr. 1995 41: 303-307.

27. Niyogi SK, Mitra U, Dutta P: Changing patterns of serotypes and antimicrobial susceptibilities of Shigella species isolated from children in Calcutta, India. J Infect Dis. 2001 54: 121-122.

28. Sack BR, Rahman M, Yunus M, Khan HE: Antimicrobial resistance in organisms causing diarrheal disease. Clin Infect Dis. 1997 24: 102-105.

29. Khan AI, Huq S, Malek MA, Hossain MI, Talukder KA, Faruque ASG et al: Shigella serotypes among hospitalized patients in urban Bangladesh and their antimicrobial resistance. Epidemiol Infect. 2004 132: 773-777.

30. Khan WA, Seas C, Dhar U, Salam MA, Bennish ML: Treatment of shigellosis: V. Comparison of azithromycin and ciprofloxacin. A double-blind,randomized, controlled trial. Ann Intern Med. 1997 126: 697-703.

31. Aggarwal P, Uppal B, Ghosh R, et al: Multi drug resistance and extended spectrum beta lactamases in clinical isolates of Shigella: a study from New Delhi, India. Tra Med Infect Dis. 201514 (4): 407-413.

32. Shakya G, Acharya J, Adhikari S, Rijal N: "Shigellosis in Nepal: 13 years review of nationwide surveillance", BMC Health Popul Nutr. 2016 35:36 